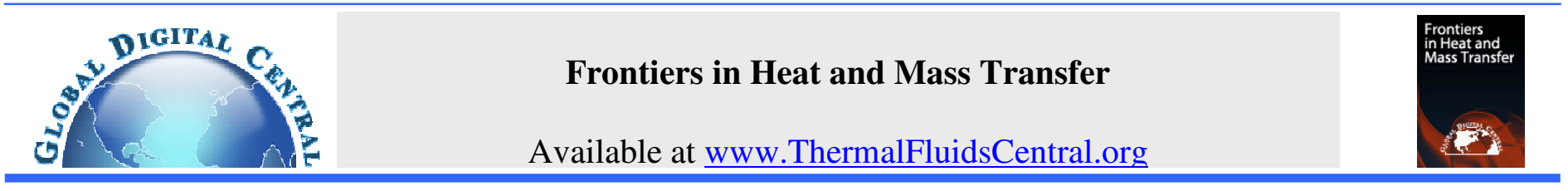

\title{
CONJUGATE HEAT TRANSFER FROM A FLAT PLATE WITH SHOWER HEAD IMPINGING JETS
}

\author{
Rajesh Kumar Panda, B.V.S.S.S. Prasad* \\ Thermal Turbomachines Laboratory, Department of Mechanical Engineering, Indian Institute of Technology, Madras, Chennai-36, India
}

\begin{abstract}
Conjugate heat transfer from a flat circular disk is investigated both computationally and experimentally with a constant heat flux imposed on its bottom surface and a shower head of air jets impinging on the top surface. The shower head consists of a central jet surrounded by four neighboring perimeter jets. Measured temperature data at twelve locations within the plate are compared with the conjugate heat transfer result obtained at the same locations computationally by Shear Stress Transport (SST) $\kappa-\omega$ turbulence model. The spacing to orifice diameter ratio $(\mathrm{H} / \mathrm{d}=1$ to 4$)$, the jet Reynolds number (7115 to 10674$)$ and the plate thickness to diameter ratio $(0.25,1$ and 2$)$ are varied. Measured temperature data within the solid plate agrees well with the computationally obtained conjugate heat transfer result. Primary peaks of the Nusselt number at the stagnation point and secondary peaks at the interaction points of the wall jets are observed. Local variation of heat transfer rate with varying $\mathrm{H} / \mathrm{d}$ is significant but found to be less sensitive to the thickness ratio. When compared with a single jet of equal mass flow rate and Reynolds number, the shower head jets provide more uniform temperature distribution with higher heat transfer rate.
\end{abstract}

Keywords: Jet impingement, Shower head, conjugate heat transfer, Experiments, CFD.

\section{INTRODUCTION}

Impinging jets are used for intense localized cooling of hot surfaces. Conventionally, a target plate of size $\mathrm{L}$ is cooled with a single jet of diameter $\mathrm{D}$ if the aspect ratio $\mathrm{L} / \mathrm{D}$ is less than 10 ; refer Fig. 1 (a). On the other hand, for a larger aspect ratio, arrays of jets arranged in either staggered or in-line fashion (Figs. 1 (b) and (c)) are used. Although heat transfer literature is replete with studies on single jet and multiple arrays of jets, a shower head as shown in Fig. 1 (d) has not been explored much. The important difference between the flow characteristics of various arrangements of impinging jets arises due to the flow resistance offered by neighboring jets. Huber and Viskanta pointed out this fact as early as 1994, in a study pertaining to array of axi-symmetric jets. Similar observations were also made later by other experimental investigators, for example, by Garimella and Schroeder (2001) in the case of nine jet impingement. It is envisaged that, as the jets are arranged in a five jet shower head fashion, (Fig. 1 (d)), the flow faces least resistance in the diagonal direction and hence the cooled target surface is expected to exhibit a more uniform temperature distribution. However, none of the earlier investigators pursued the flow and heat transfer study with the shower head arrangement. It is therefore proposed in the present paper that a shower head, consisting of one central jet and four perimeter jets shall replace the single jet by distributing the same mass flow rate through the five jets. The aspect ratio based on the pitch circle diameter of the perimeter jets $(l / \mathrm{L})$, can be chosen to have a wider coverage of the target plate by the impinging jets.

One of the most recent experimental local heat transfer study of jet impingement on a flat surface is by Sagot et al (2007). They compared the computational results obtained by various authors who used different RANS models, and recommended the usage of $\kappa-\omega$ SST turbulence model. Similar recommendation was also made by Ramakumar and Prasad (2008) for a concave curved surface. Sagot et al
(2007) noted that the maximum difference in Nusselt number values calculated with constant heat flux and constant temperature boundary conditions was $12 \%$. Katti and Prabhu (2007) reported local Nusselt number variation by imposing constant heat flux condition on a very thin foil plate, where the heat conduction could be neglected. Likewise, a large number of frequently referred jet impingement investigations on single jet by Martin (1977), Jambunathan et al. (1993), Naitoh et al. (2001) and O'Donovon (2007) and on multiple jets by Viskanta (1993), Emmanuel et al. (2000), Geers (2008) were reported. In none of these, the heat conduction within the plate was considered. However, in practice, the thermal boundary conditions cannot be simply specified as constant temperature or constant heat flux, because the impingement heat transfer takes place together with heat conduction in the plate. Thus, this is strictly a conjugate problem. It is evident from the study of Luikov (1973) and Prasad and Sarkar (1993), that thermal conductivity and thickness ratios dictate whether the conjugate study is important or not. In order to address this aspect, Siba et al. (2003) constructed an experimental model with $12.7 \mathrm{~mm}$ thick stainless-steel plate and used inverse heat conduction technique to calculate both surface temperature and heat flux by feeding measured temperature at $0.76 \mathrm{~mm}$ below the top surface. With the objective of studying the conjugate heat transfer, Yang and Tsai (2007) used low Reynolds k- $\omega$ turbulence model to predict the heat transfer coefficient for the flat plate model of Siba et al. (2003). They predicted top (impingement) surface temperature variation by imposing different uniform heat flux values at the bottom surface.

All the reported investigations in the foregoing papers pertain only to the single jet or in-line/staggered arrays. It appears that there are only two investigation reported on the shower head arrangement. Aldabbagh and Sezai (2002) reported a study of laminar jets emanating from square holes with isothermal boundary condition imposed on the impingement plate. The second one is by the present authors, Panda et al. (2010). 


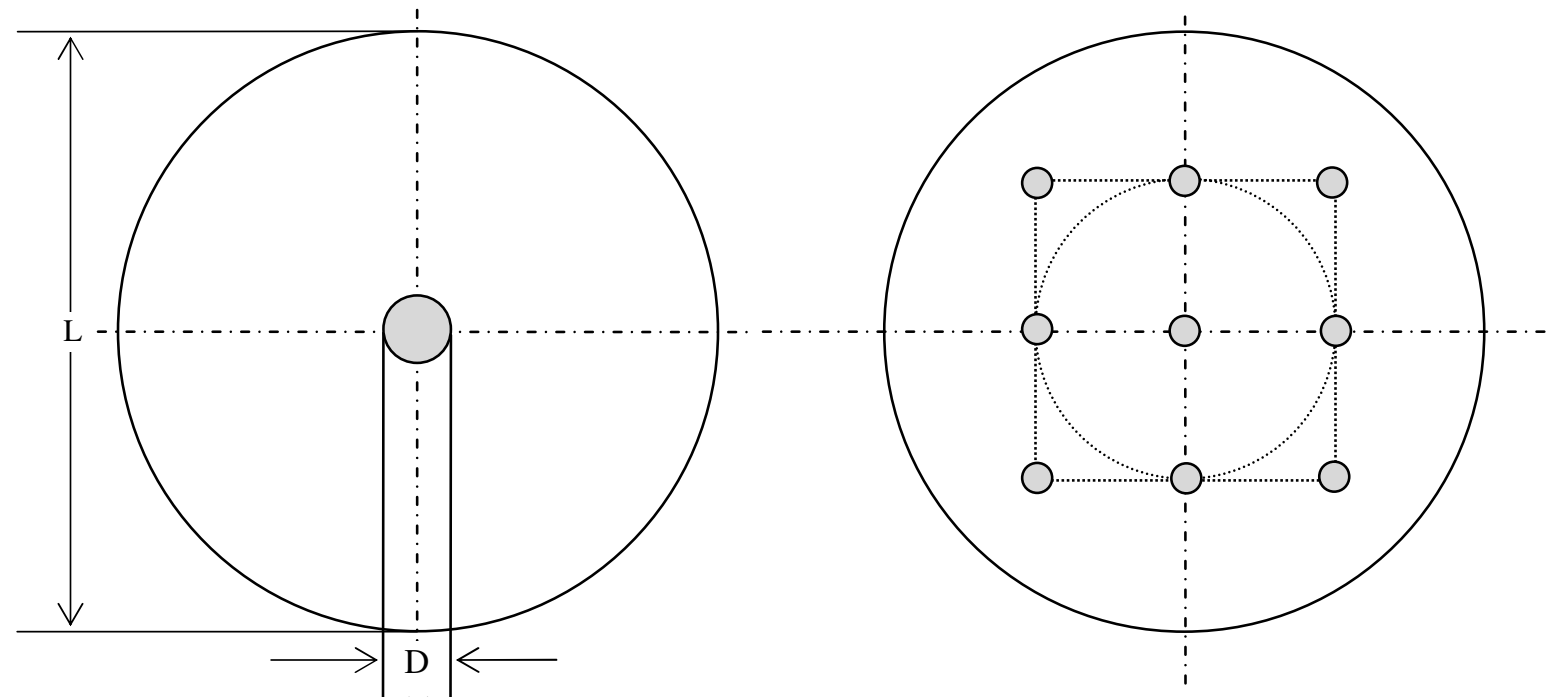

(a)

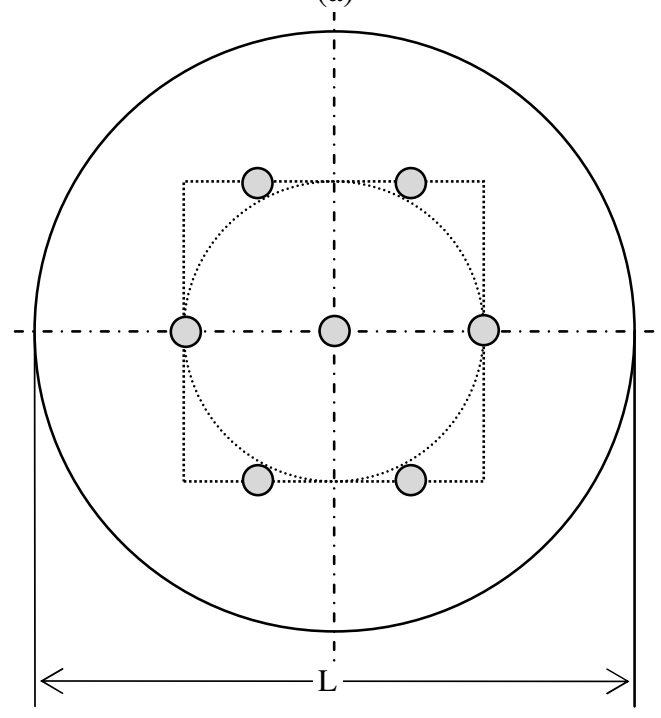

(c)

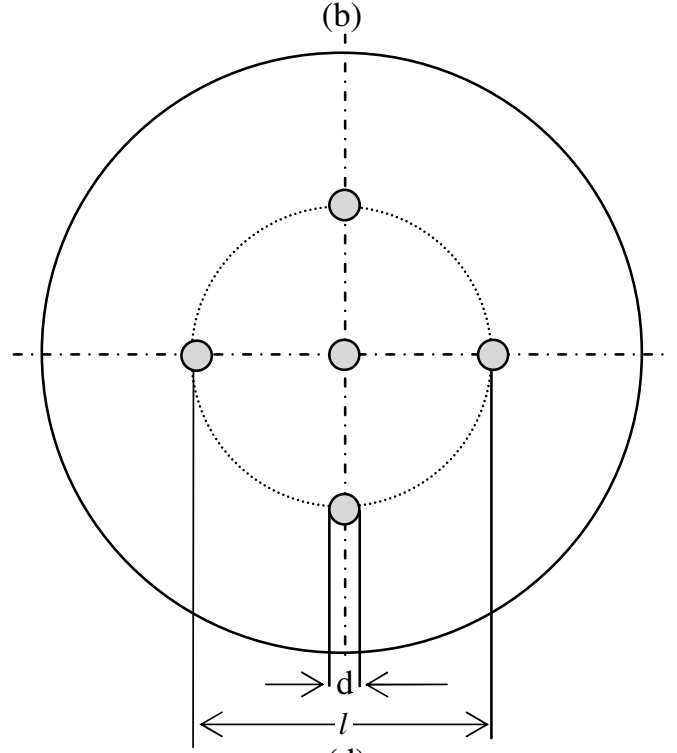

(d)

Fig.1 Arrangement of impinging jets, (a) single jet, (b) in-line, (c) staggered and (d) shower head.

It is evident from the above literature that the computational and experimental conjugate impingement heat transfer studies on the five jet turbulent shower head configuration have not been widely investigated. The broad objectives of the paper are therefore to report for the shower head geometry, (i) the validation of conjugate heat transfer computation by comparing with the measured temperature across the thickness of the plate, (ii) the flow structure of the interacting jets, (iii) the effect of varying H/d and Reynolds number on heat transfer rate, and (iv) a typical case of comparison between heat transfer rates from the single jet and the shower head.

\section{EXPERIMENTAL DETAILS}

The schematic layout of the experimental set up is shown in Fig.2. Air is supplied by a reciprocating air compressor through a calibrated rotameter. Air filter and pressure regulator are installed upstream of the rotameter to filter the air and to maintain the downstream pressure. Air filter removes the moisture and dust particles from the incoming air. Flow regulating and bypass valves are placed in supply line to control the flow rate. Air enters into the plenum chamber through diverging section to reduce the velocity of the air. A seamless cylinder of $260 \mathrm{~mm}$ diameter, $500 \mathrm{~mm}$ height and $10 \mathrm{~mm}$ thickness, is used as plenum chamber, whose size is large enough to stabilize the flow and reduce the fluctuations. Air from plenum chamber enters into the impingement chamber which is made of $160 \mathrm{~mm}$ diameter, $250 \mathrm{~mm}$ height and $4 \mathrm{~mm}$ thick mild steel cylinder. Air ejects as jet through five, $10 \mathrm{~mm}$ diameter orifice and impinges on the target surface. Four holes are placed $90^{\circ}$ apart from each other around a central hole and on the perimeter of a pitch circle, shown in Fig. 3 (a). Distance between the centre hole and perimeter holes hole is $40 \mathrm{~mm}$. The impingement plate $(160 \mathrm{~mm}$ diameter and $20 \mathrm{~mm}$ thickness) is heated from the bottom surface and circumferentially insulated. The plate is made up of mild steel. Impingement plate and heating element are placed over a stand, whose height can be varied by a lead screw mechanism to obtain the required $\mathrm{H} / \mathrm{d}$. The power input to the heating coil is controlled by a variable auto-transformer and measured by precision voltmeter and ammeters.

The impingement plate is instrumented with pre-calibrated T-type thermocouples placed $30^{\circ}$ apart from each other at different depth and different elevations to obtain temperature distribution across the thickness of the plate. Positions of thermocouples are shown in Fig. 3 (b) and Table 1. Overall diameter of the thermocouple is $1 \mathrm{~mm}$. Agilent 34970A (HP make) and personal computer with required software are used for data acquisition and storage. The experimental uncertainty is estimated to be about $6 \%$. 


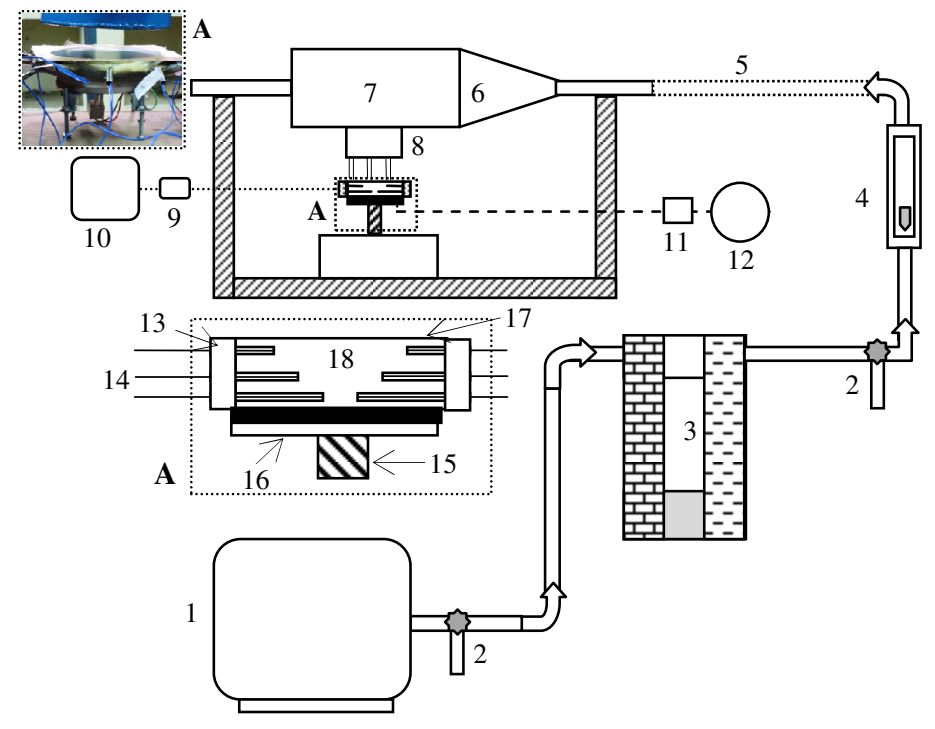

Fig. 2 Schematic diagram of experimental set-up: A. Test section 1. Compressor, 2. Bypass line with control valve 3. Air drier and filter, 4. Rotameter, 5. Flexible pipe, 6. Diverging section, 7. Plenum chamber, 8. Impingement chamber, 9. Data acquisition system, 10. Personal computer, 11. Ammeter, 12. Variable auto transfer, 13. Insulation, 14. Thermocouple wire, 15. Lead screw arrangement, 16. Heating arrangement, 17. Target surface, 18. Mild steel plate

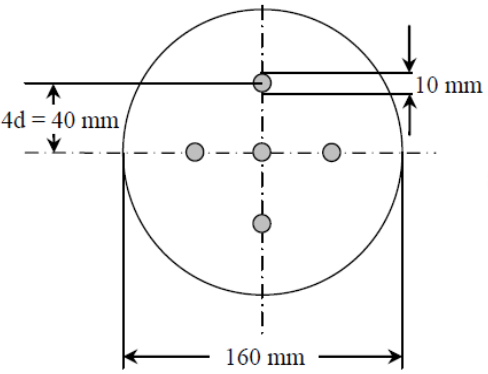

(a)

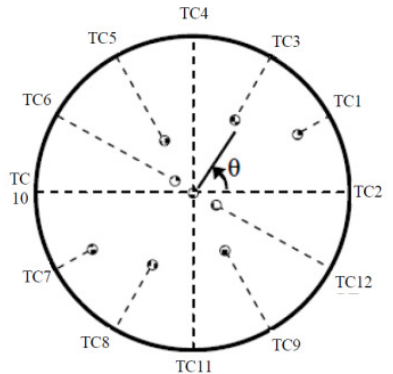

(b)
Fig. 3 Schematic diagram of, (a) semi-confining plate and (b) Impingement plate

Table 1. Positions of thermocouple

\begin{tabular}{|c|c|c|c|}
\hline Thermocouple number & $\mathrm{h}, \mathrm{mm}$ & $\mathrm{R}, \mathrm{mm}$ & Angle, $\theta^{\mathbf{}}$ \\
\hline TC1 & 17 & 65 & 30 \\
\hline TC2 & 15 & 0 & 0 \\
\hline TC3 & 13.5 & 50 & 60 \\
\hline TC4 & 12 & 0 & 90 \\
\hline TC5 & 9.5 & 35 & 120 \\
\hline TC6 & 6 & 20 & 150 \\
\hline TC7 & 17 & 65 & 210 \\
\hline TC8 & 13.5 & 50 & 240 \\
\hline TC9 & 9.5 & 35 & 300 \\
\hline TC10 & 9 & 0 & 180 \\
\hline TC11 & 6 & 0 & 270 \\
\hline TC12 & 6 & 20 & 330 \\
\hline
\end{tabular}

\section{COMPUTATIONAL METHODOLOGY}

Figure 4 shows the geometric model that mimics the physical model used for experiment of interest. It consists of a semi-confined circular plate and $160 \mathrm{~mm}$ diameter, $20 \mathrm{~mm}$ thick impingement plate. The semiconfining plate consists of five orifices each of them is $10 \mathrm{~mm}$. The jet ejects from the circular orifice to impinge on the solid plate. Distance between the impingement plate and the top wall is so varied as to obtain the $\mathrm{H} / \mathrm{d}$ ratio from 1 to 4 .

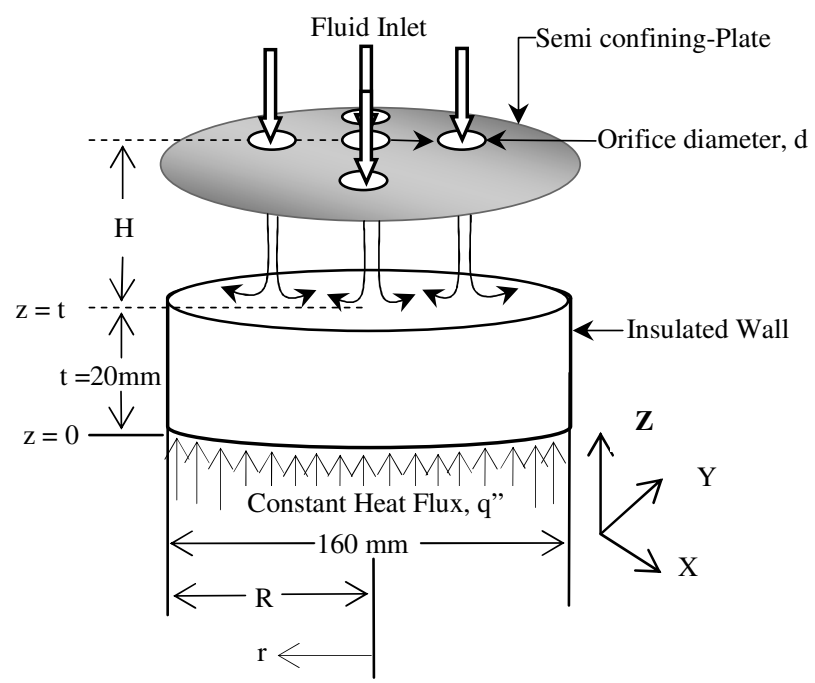

Fig. 4 Schematic of the geometry and boundary conditions

The model can be divided into the fluid zone and the solid zone. The computational mesh suitable for finite volume method is generated by automatic grid generating tool GAMBIT 2.3.16. Non-uniform grids with clustered nodes are generated in the regions where steep gradient of temperature is expected. Optimum grid size is selected after the grid independence study. The grid sizes finally chosen are: 1.8 million for $\mathrm{H} / \mathrm{d}=1 ; 2.3$ million for $\mathrm{H} / \mathrm{d}=2$; and 2.7 million for $\mathrm{H} / \mathrm{d}=4$. The values of maximum $\mathrm{y}+$ used in the present computations are around 1 . Figure 5 shows the mesh used for $\mathrm{H} / \mathrm{d}=4$.
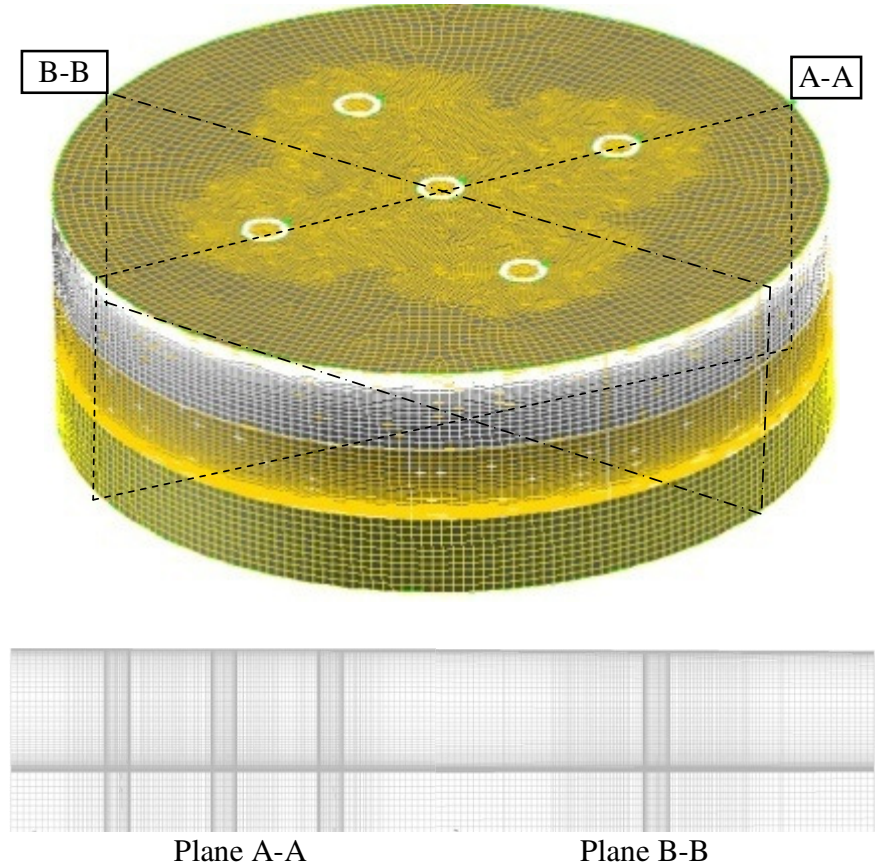

Fig. 5 Mesh used for $\mathrm{H} / \mathrm{d}=4$ 
For the purpose of computation, a specified velocity inlet condition is imposed at the entry to the orifices. A free stream turbulence intensity of $6 \%$ is chosen at the exit of orifice. The jet after hitting the plate exits into the atmosphere in transverse direction, all around the plate periphery, where constant (ambient) pressure outlet condition is imposed. A constant heat flux value is specified at the bottom surface of the plate and the periphery is circumferentially insulated. All the input values are those used in experiments.

The three dimensional conjugate heat transfer problem is formulated with the following assumptions: (i) the fluid is incompressible, (ii) the fluid properties are constant (iii) radiation and natural convection are neglected, (iv) viscous dissipation is absent and (v) the flow is steady. The governing equations used for simulations are the Reynolds averaged continuity, momentum and the energy equations along with the equations for modeling the turbulence quantities.

Boundary conditions used for the present study are,

i) No slip boundary condition

$$
\mathrm{V}_{\mathrm{w}}=0 \text {, }
$$$$
\text { at } \mathrm{z}=\mathrm{t}
$$

ii) Flow inlet condition,

$$
\begin{aligned}
& V_{r}, V_{\theta}=0, V_{z}=V_{o e} \\
& \text { and Toe }=300 \mathrm{~K}
\end{aligned}
$$

iii) Entrainment condition,

$$
\begin{aligned}
\mathrm{P} & =\mathrm{P}_{\text {atm }} \\
\text { at } \mathrm{r} & =\mathrm{R}, 0<\mathrm{z}<\mathrm{H}
\end{aligned}
$$

iv) Conjugate boundary condition,

$$
\begin{aligned}
\mathrm{T}_{\mathrm{s}} & =\mathrm{T}_{\mathrm{f}}, \\
k_{s} \frac{\partial T_{s}}{\partial Z} & =k_{f} \frac{\partial T_{f}}{\partial Z}, \\
\text { at } \mathrm{z}=\mathrm{t} &
\end{aligned}
$$

v) Constant heat flux condition,

$$
\begin{aligned}
& -\mathrm{k}_{\mathrm{s}} \frac{\partial \mathrm{T}_{\mathrm{s}}}{\partial \mathrm{z}}=\mathrm{q}^{\prime \prime} \\
& \text { at } \mathrm{z}=0,0<\mathrm{r}<\mathrm{R}
\end{aligned}
$$

vi) Adiabatic condition,

$$
\begin{gathered}
\frac{\partial T_{s}}{\partial r}=0, \\
\text { at } \mathrm{r}=\mathrm{R}, 0 \leq \mathrm{z} \leq \mathrm{t},
\end{gathered}
$$

A finite volume based solver Fluent 6.3 is used for solving the governing continuity, momentum, energy and turbulence model equations. SIMPLE algorithm is used for pressure velocity coupling. The solution is considered to be converged when the residual is in the order of 10-4 for continuity, momentum and turbulence equations and $10-6$ for energy equation. The $\kappa-\omega$ SST model is adopted for simulation. Further, area weighted average of temperature of the impingement surface is continuously monitored, so that the variation will be within $0.1 \%$ for 1000 consecutive iterations. Simulation continued till solid plate reaches steady state.

The parameters investigated in the present study include (i) effect of $\mathrm{H} / \mathrm{d}$, varied as 1,2 and 4, (ii) effect of jet exit Reynolds number, varied as 7115, 8299, 9500 and 10672 (iii) thickness of the plate is varied as $20 \mathrm{~mm}, 10 \mathrm{~mm}$ and $2.5 \mathrm{~mm}$. Diameter of the jet orifice is kept constant as $10 \mathrm{~mm}$.

\section{RESULTS AND DISCUSSION}

\subsection{Validation of Conjugate Heat Transfer}

As the fluid jet ejects out of the orifice with uniform velocity profile into the quiescent ambient, a continuous reduction in velocity takes place from its centre line to the outer boundary. With increasing distance from the exit, and increasing momentum exchange between jet and the ambient the free boundary of the jet is broadens while the potential core contracts. On the impingement surface the wall jets are formed and spread radially. The wall jet emanating from each impinging jet forms a collision front due to its interaction with the neighbors. Consequently an up-wash fountain flow takes place. Figure 6 shows these characteristic features of the shower head impinging jet along the chosen planes A-A (taken through three jets) and $\mathrm{B}-\mathrm{B}$ and (plane considering centre jet and in between two perimeter jets) for $\mathrm{H} / \mathrm{d}=4$.

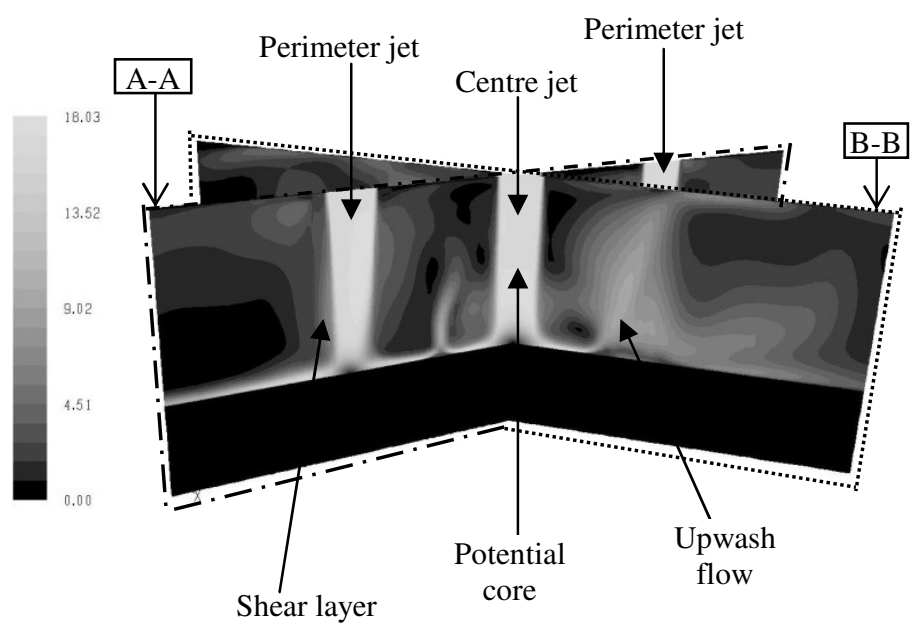

Fig.6 Velocity contour, $\mathrm{H} / \mathrm{d}=4$ and $\mathrm{Re}=9500$

The heat transfer between the solid plate and the jet is determined by the condition of continuity of heat flux (conjugate condition) at the interface. The validity of the computational model for the conjugate heat transfer problem is ascertained by comparing the numerical results with the measured temperature data. Figure 7 shows the computationally obtained distribution of differential temperature (T$\mathrm{T}_{\min }$ ) value is plotted at various heights from the bottom surface with the corresponding measured value superimposed on the same figure. Minimum temperature is measured along TC1 i.e. at the centre of the line drawn at a distance of $17 \mathrm{~mm}$ from bottom surface. Considering the error band of $\pm 6 \%$, calculated using the uncertainity analysis the agreement between the measured and computational data is found to be good, lending confidence to the overall computational procedure.

Although all the plates are heated at their bottom surface by the supplying a constant heat flux; the top surface heat flux shows a different spatial variations of temperature (Fig.8) and heat flux (Fig.9) because of the variation of flow structure.

Figure 8 shows the temperature distribution along line A-A on the top and bottom surfaces of three plates of different thickness values $2.5 \mathrm{~mm}, 10 \mathrm{~mm}$ and $20 \mathrm{~mm}$ along line A-A. It is observed that in case of $2.5 \mathrm{~mm}$ thick plate the values of temperature and its radial variation is almost the same on both the top and bottom surfaces. However in case of $10 \mathrm{~mm}$ thick plate the temperature difference on the top surface is larger than the bottom surface. This temperature difference increases further for the $20 \mathrm{~mm}$ thick plate. Notice that as the thickness increases, the both top and bottom surface attain near isothermality. 

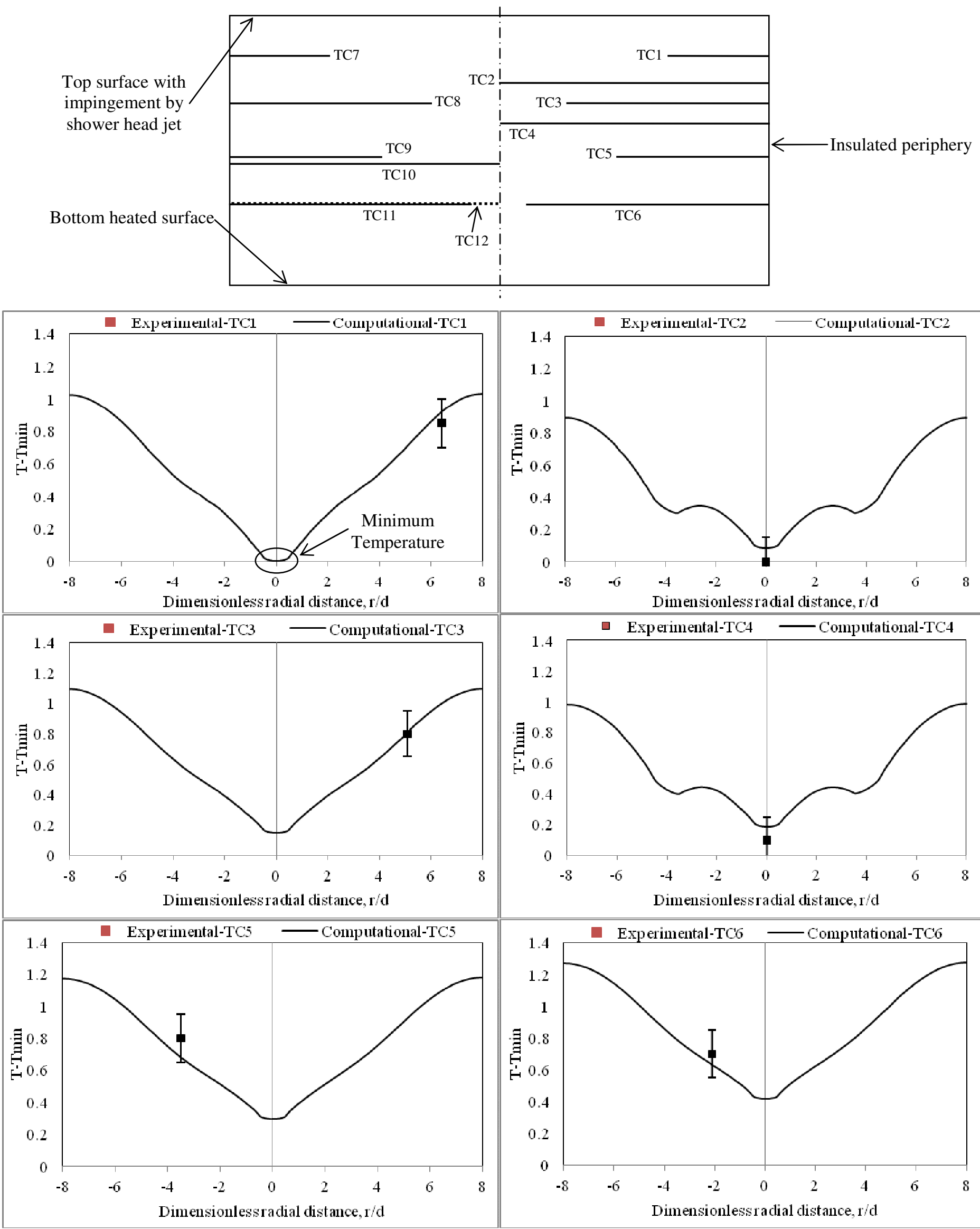

Fig. 7 Comparison of computed differential temperature distribution with the measured Value in the solid plate for $\mathrm{Re}=10674, \mathrm{H} / \mathrm{d}=2$ and q" $=3000 \mathrm{~W} / \mathrm{m}^{2} . \mathrm{K}$ 


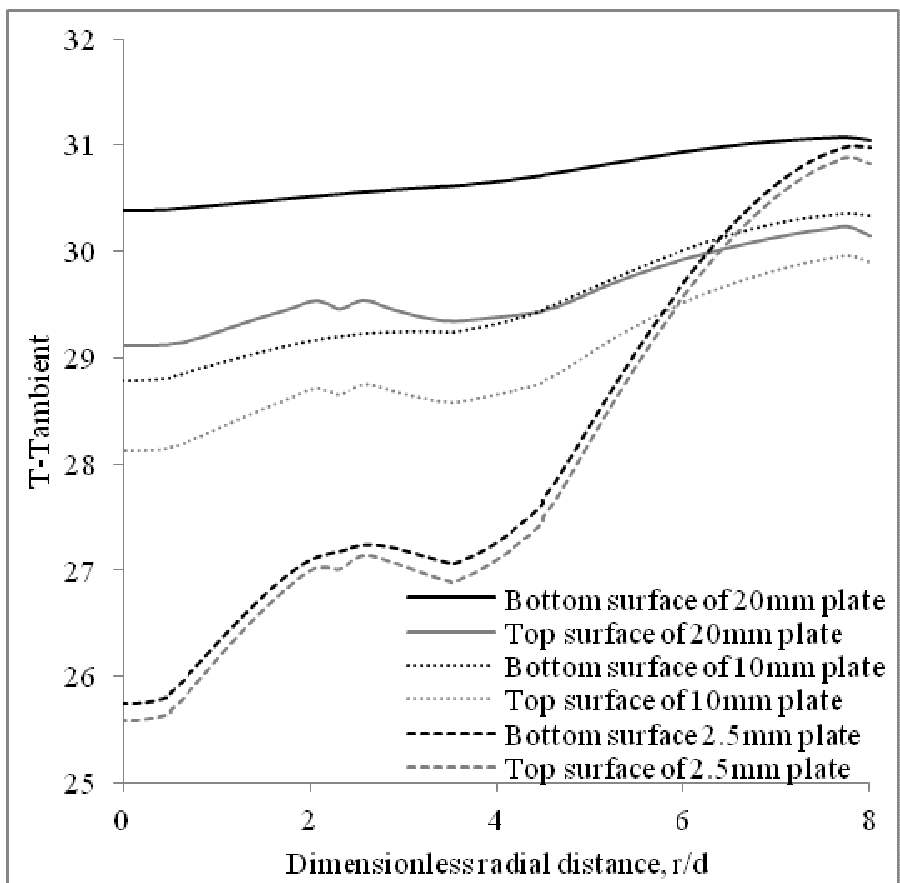

Fig. 8 Temperature distribution on plane A-A; $\mathrm{H} / \mathrm{d}=2, \mathrm{q}{ }^{\prime}=3000 \mathrm{~W} / \mathrm{m}^{2}$ and $\mathrm{Re}=9500$

Figure 9 shows the steady state heat flux distribution on the top and bottom surfaces of the three plates along line A-A. The radial variation of heat flux on the top surface is qualitatively the same for all three plates. The differences among them are attributed to the effect of conjugation. In a given radial variation, the heat flux values are larger where cooling rates are higher, e.g. at the stagnation points and the interaction zones. The peaks and valleys of the surface heat flux variation are explainable by the flow structure and discussed in the next section.

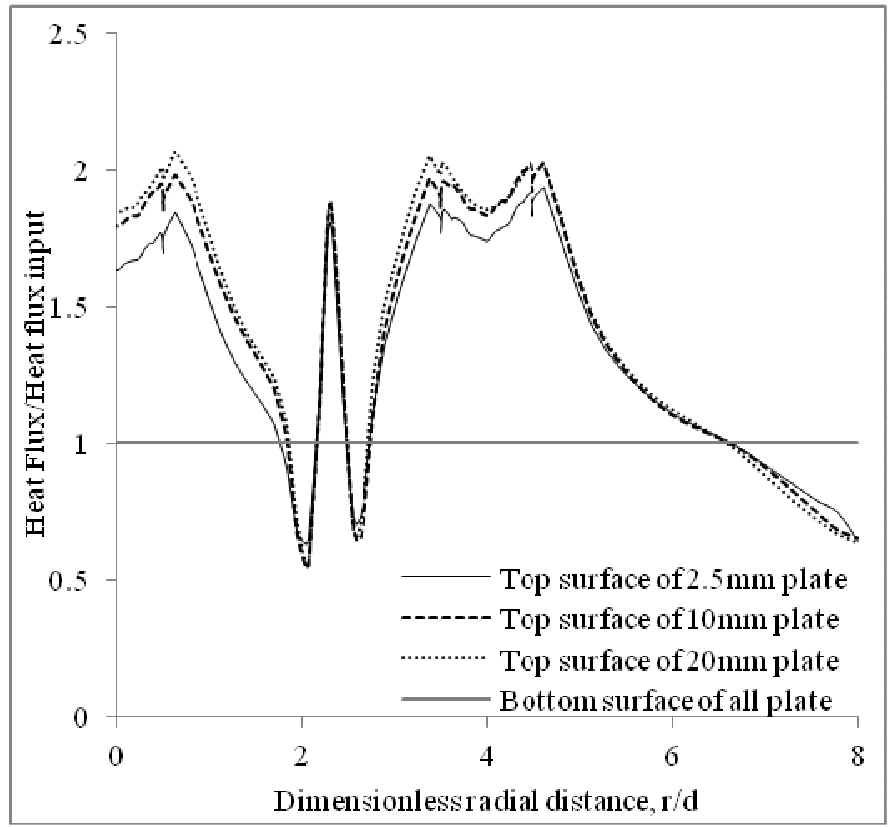

Fig.9 Heat flux distribution on plane A-A; for $\mathrm{H} / \mathrm{d}=2, \mathrm{q} "=3000 \mathrm{~W} / \mathrm{m}^{2}$ and $\mathrm{Re}=9500$

Defining Nusselt number $\mathrm{Nu}=(\mathrm{h} d) / \mathrm{k}$, where heat transfer coefficient: $h=q " /\left(T_{w}-T_{o e}\right)$, the variation of Nusselt number is presented in Fig. 10 for the three plates along line A-A. A variation of around $6 \%$ is observed in the local Nusselt number value along A-A between $2.5 \mathrm{~mm}$ and $20 \mathrm{~mm}$ thick plates. The typical area weighted average heat transfer coefficients varies between 99.8 to $101 \mathrm{~W} / \mathrm{m}^{2} \mathrm{~K}$ for plate of thickness 2.5 and $20 \mathrm{~mm}$ respectively, variation is near $1 \%$.

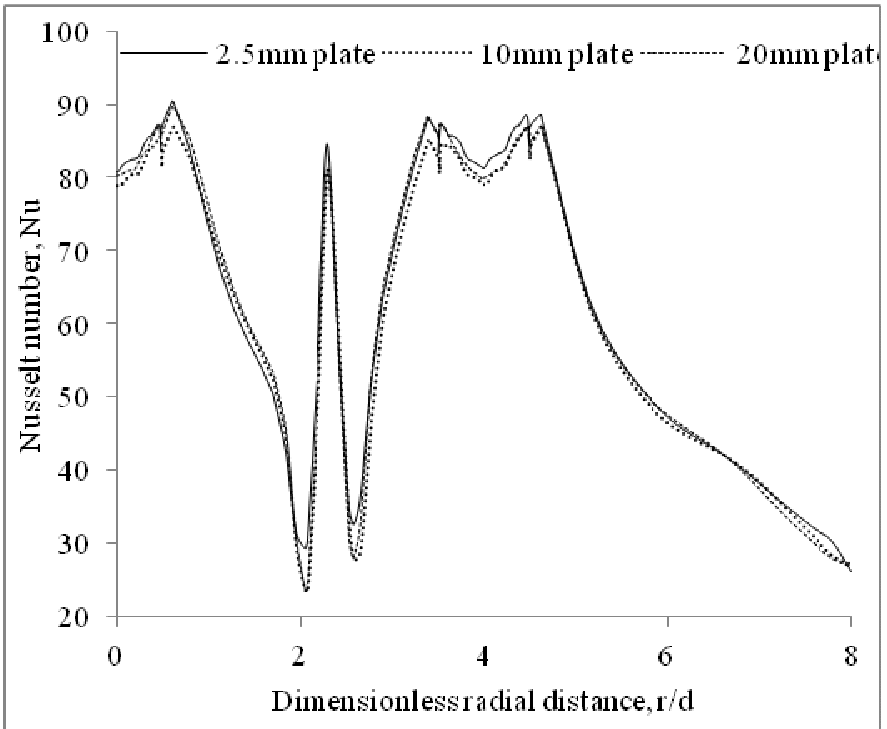

Fig. 10 Nusselt number distribution on plane A-A; for $\mathrm{H} / \mathrm{d}=2, \mathrm{q} "=3000 \mathrm{~W} / \mathrm{m}^{2}$ and $\mathrm{Re}=9500$

Figure 11 illustrates the dependence of Nusselt number on Reynolds number for $\mathrm{H} / \mathrm{d}=2$ and q" $=2000 \mathrm{~W} / \mathrm{m}^{2}$ on line A-A. As expected the heat transfer rate is higher for high Reynolds number. At any Reynolds number the heat transfer rate has the highest value at the stagnation region and reduces radially. Second peaks are observed at the secondary stagnation points near upwash region. As stated earlier the position of second peak in the Nusselt number is independent of the Reynolds number hence the positions of the secondary peaks are found close to each other. Figure 12 shows the variation of Nusselt number with different Reynolds number in line B-B. The difference between the Nusselt number behaviors along line A-A and B-B is that, the magnitude of second peak is lower along line B-B. This may be because interaction at the nodal point of separation is weaker than secondary stagnation zone. There is a significant variation in heat transfer coefficient with Reynolds number. Area weighted average heat transfer coefficient for the $\mathrm{Re}=7115$ is $85.3 \mathrm{~W} / \mathrm{m}^{2} \mathrm{~K}$ and for the $\mathrm{Re}=10674$ case is $125.3 \mathrm{~W} / \mathrm{m}^{2} \mathrm{~K}$ over the impingement surface area. In other words the Nusselt number variation is dependent on the flow structure which in turn is dependent on various factors, including Reynolds number.

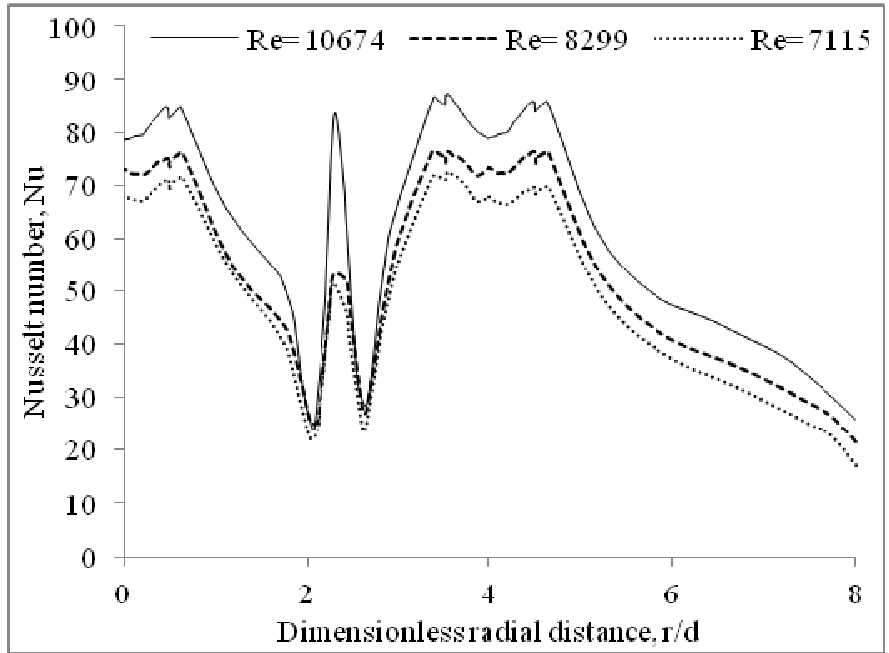

Fig. 11 Nusselt number distribution on plane A-A; for $\mathrm{H} / \mathrm{d}=2$ and $\mathrm{q}=3000 \mathrm{~W} / \mathrm{m}^{2}$. $\mathrm{K}$ 


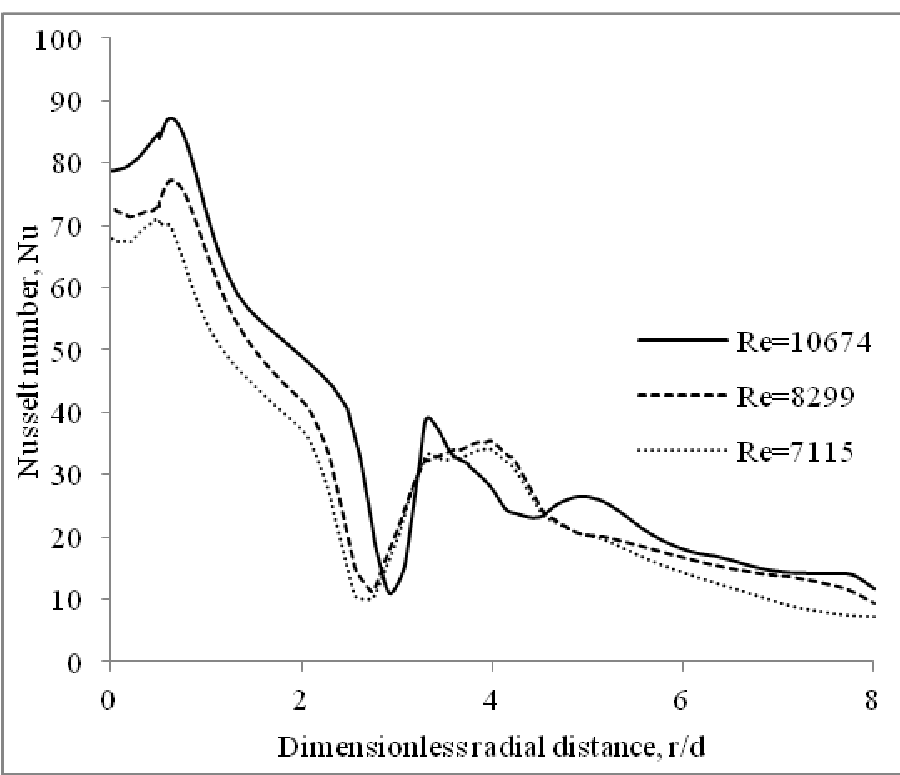

Fig. 12 Nusselt number distribution on plane B-B; for $\mathrm{H} / \mathrm{d}=2$ and $\mathrm{q} "=3000 \mathrm{~W} / \mathrm{m}^{2}$. K

Three important observations that emerged from this conjugate calculation are as follows:

(i) The surface temperature variation differs significantly with the plate thickness.

(ii) The individual surface heat flux variations are highly significant and primarily dependent on flow structure rather than thickness.

(iii) The Nusselt number variation is therefore necessarily dependent both on thickness as well as flow structure.
The Nusselt number result (Fig. 11) does not seem to corroborate this result, because of the apparent insignificant variation of Nusselt number with thickness at a given Reynolds number. This may give an impression that conjugate heat transfer calculations are not required. This is not a correct conclusion due to the following reason, let assume that had the calculations are performed, with a constant heat flux boundary condition on the top surface, ignoring the effect of conduction, the Nusselt number variation would have been almost the same but the temperature of the surface would have shown wide variations with peaks and valleys. This is contrary to the fact where the surface temperature of a thick plate is more uniform as in Fig.8. Thus the result is clearly indicates the importance of conjugate heat transfer and it heartening to notice that the computation predict the value quite well.

\subsection{Effect of plate spacing to jet diameter ratio (H/d)}

\section{2. 1. Flow structure}

The wall jets separates from each other in the impingement plane due to their interaction, are shown in Fig.13 for three different $\mathrm{H} / \mathrm{d}$ values. When $\mathrm{H} / \mathrm{d}$ is lower $(\mathrm{H} / \mathrm{d}=1)$ the separated flow/upwash flow impinges on the top wall to form low velocity wall jet. The top wall jet is then entrained into the main jet stream leading to the formation of two counter rotating vortices, shown in Fig. 13 (a). When $\mathrm{H} / \mathrm{d}$ is larger $(\mathrm{H} / \mathrm{d}=2$ and 4$)$ however the upwash flow cannot reach the top plate. As a result the upper wall jets do not form for higher values of $\mathrm{H} / \mathrm{d}$, as shown in Fig.13 (b and c). The upwash fountain forms at the interaction region between the centre jet and the perimeter jet. Another pair of ring shaped eddies called as wall eddies are formed between the counter rotating vortex and the wall, as shown in Fig.13 (d).
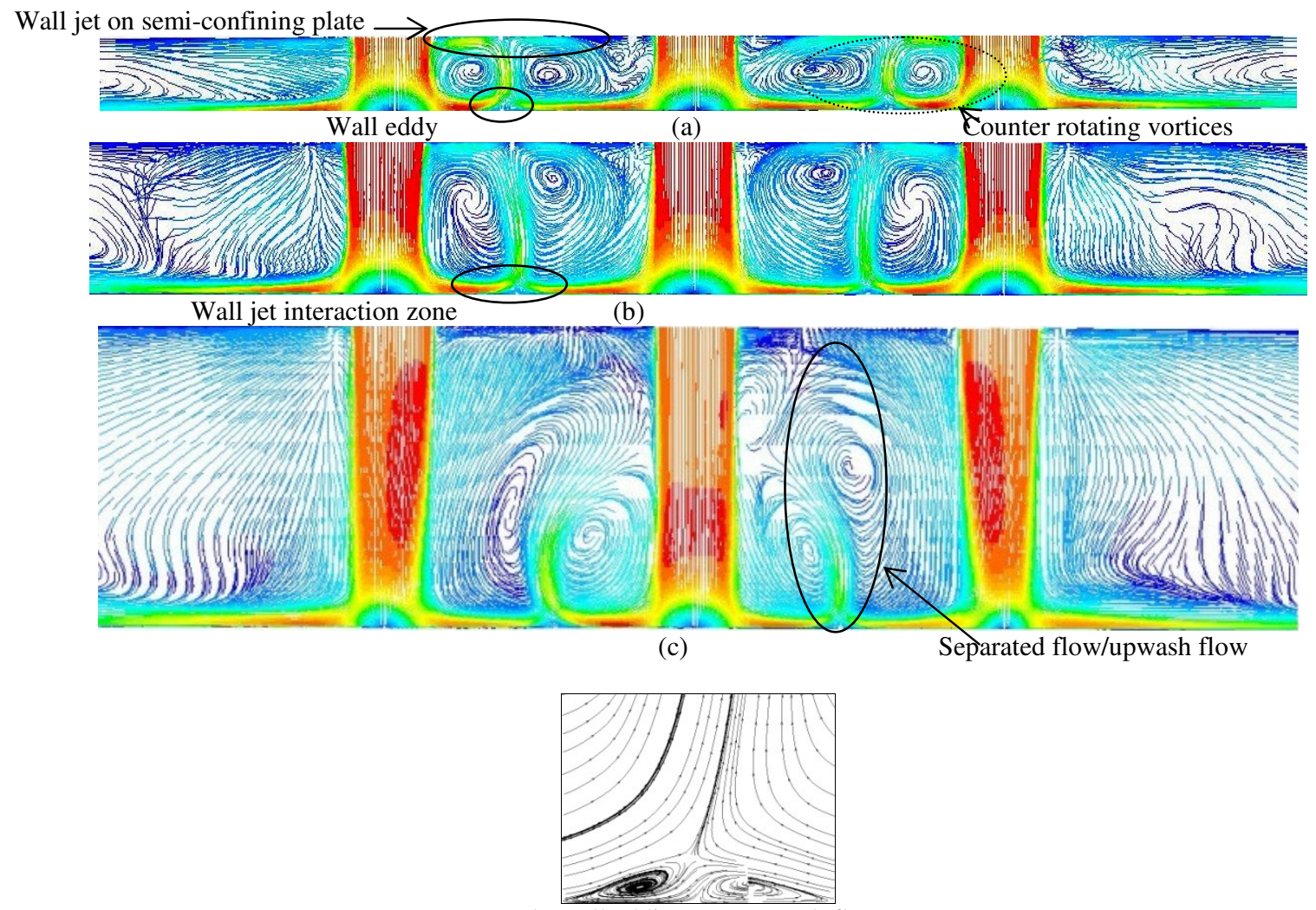

(d) Wall eddies near upwash flow

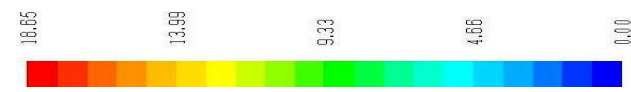

Fig. 13 Path line colored by velocity magnitude in plane A-A, (a) $H / d=1$, (b) $H / d=2$ and (c) $H / d=4 ; R e=9500$ 
Figure 14 shows the flow structure along plane $B-B$ for $H / d=1,2$ and 4 . In case of $\mathrm{H} / \mathrm{d}=1$ the flow smoothly spreads radially outward, as shown in Fig. 14 (a). Whereas in case of $\mathrm{H} / \mathrm{d}=2$ and 4 flow separates and lifts up at the interaction region of wall jets, shown in Fig. 14 (b) and (c). Due to which there is a formation of vortex in plane B-B in between nodal point of separation and centre jet for $\mathrm{H} / \mathrm{d}=2$ and 4 .

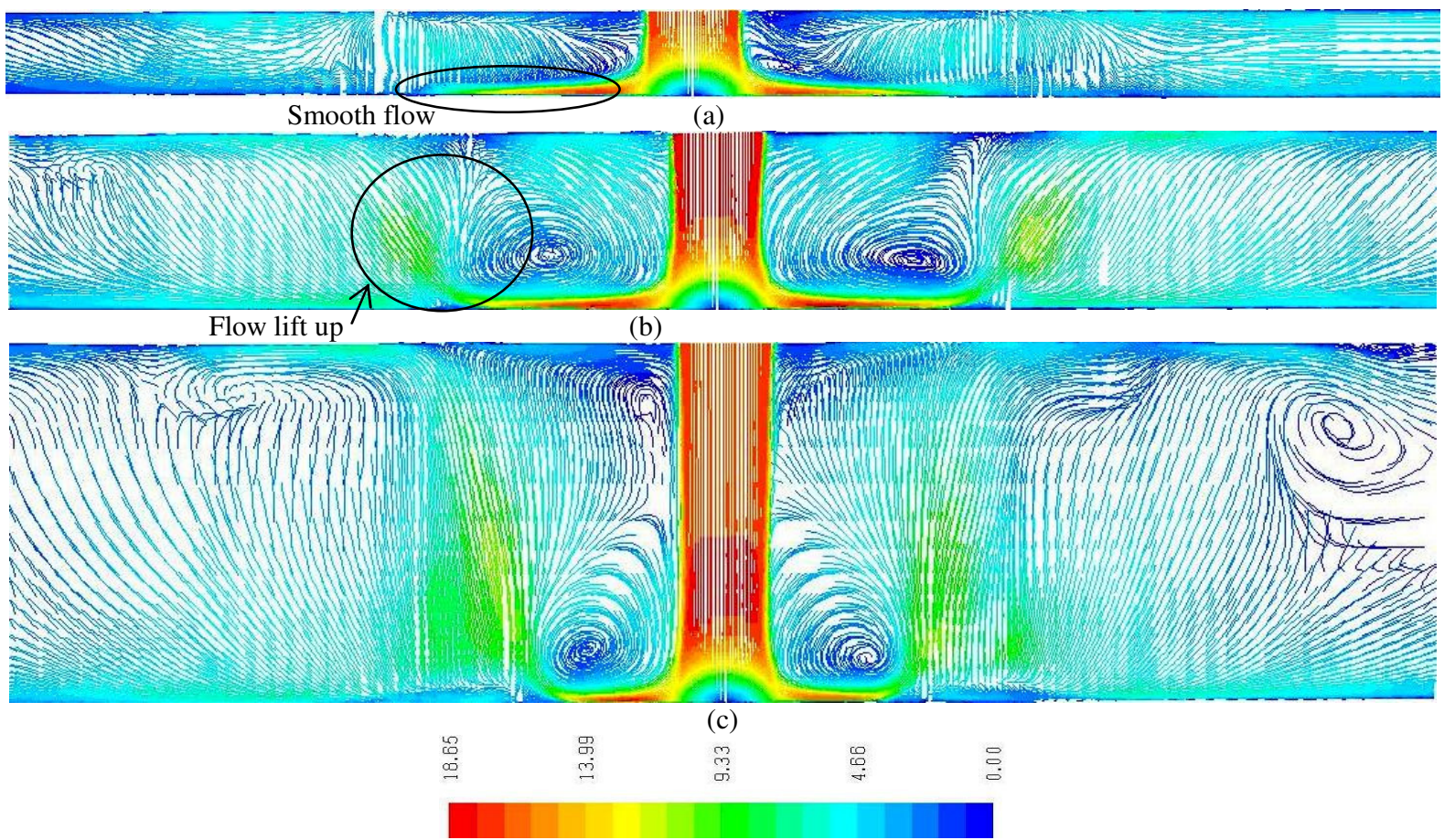

Fig. 14 Path line colored by velocity magnitude in plane $B-B$, (a) $H / d=1$, (b) $H / d=2$ and (c) $H / d=4 ; R e=9500$

\section{2. 2. Heat Transfer}

Figure 15 shows the variation of Nusselt number with different plate spacing to jet diameter ratio for $\mathrm{Re}=9500$ and $\mathrm{q} "=3000 \mathrm{~W} / \mathrm{m}^{2}$ in line A-A. For higher H/d $(=4)$, the maximum Nusselt number value in the stagnation region is lower compared to lower $\mathrm{H} / \mathrm{d}(=1)$ by about $6-7 \%$. The difference among Nusselt numbers at the centre jet is less compared to perimeter jet, because the perimeter jets are more affected by quiescent ambient air. The secondary peaks are observed at the interaction region of the wall jets of centre and perimeter jet. The position of the second peak point corresponds to the position of the formation of wall eddies and the valleys corresponds to flow separation point. The upwash fountain for lower $\mathrm{H} / \mathrm{d}$ is more prominent hence larger secondary peaks are observed for lower $\mathrm{H} / \mathrm{d}$.

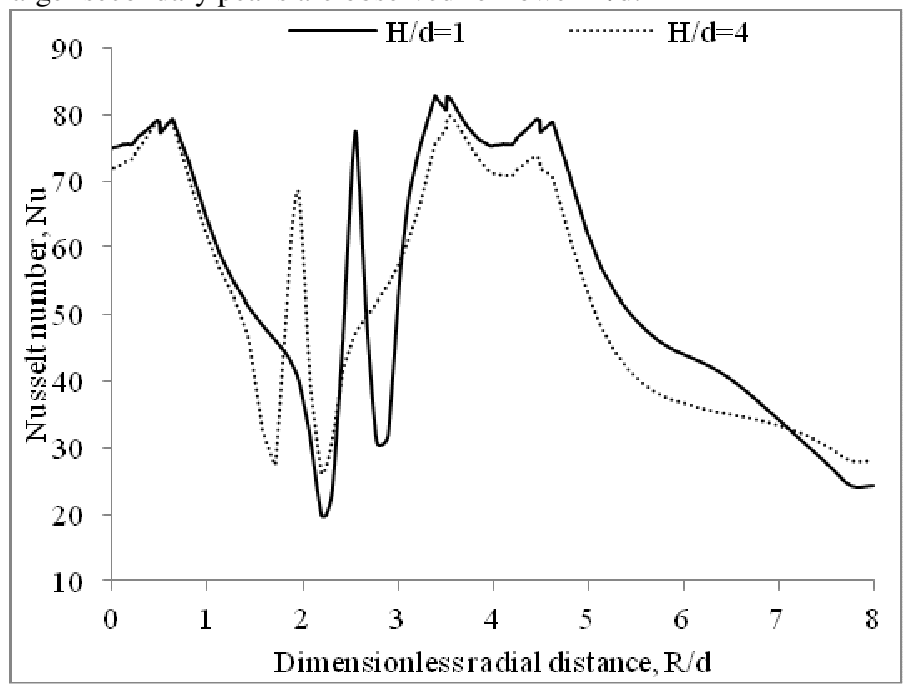

Fig.15 Nusselt number distribution on plane A-A for varying $\mathrm{H} / \mathrm{d}, \mathrm{q}{ }^{\prime}=3000 \mathrm{~W} / \mathrm{m}^{2}$ and $\mathrm{Re}=9500$
Figure 16 shows the variation of Nusselt number with different $\mathrm{H} / \mathrm{d}$ for same condition in line B-B. Here the heat transfer behavior in the stagnation zone is similar as in line A-A. A peak in the $\mathrm{Nu}$ is observed for $\mathrm{H} / \mathrm{d}=4$ in the radial direction whereas due to smooth flow in case $\mathrm{H} / \mathrm{d}=1$ no such peak is observed. The position of peak depends upon the position of interaction of wall jets. The wall eddies induces consecutively by the large scale toroidal vortices hits the plate, which are responsible for the enhancement of local heat transfer.

Area weighted average heat transfer coefficient for the $\mathrm{H} / \mathrm{d}=4$ is 97.4 W/m $/ \mathrm{m}^{2} \mathrm{~K}$ and for the $\mathrm{H} / \mathrm{d}=1$ case is $100.8 \mathrm{~W} / \mathrm{m}^{2} \mathrm{~K}$ over the impingement surface area. In other words, although there are significant local variations of Nusselt number, the average value differs only by 3 $4 \%$ as $\mathrm{H} / \mathrm{d}$ varies from 1 to 4 .

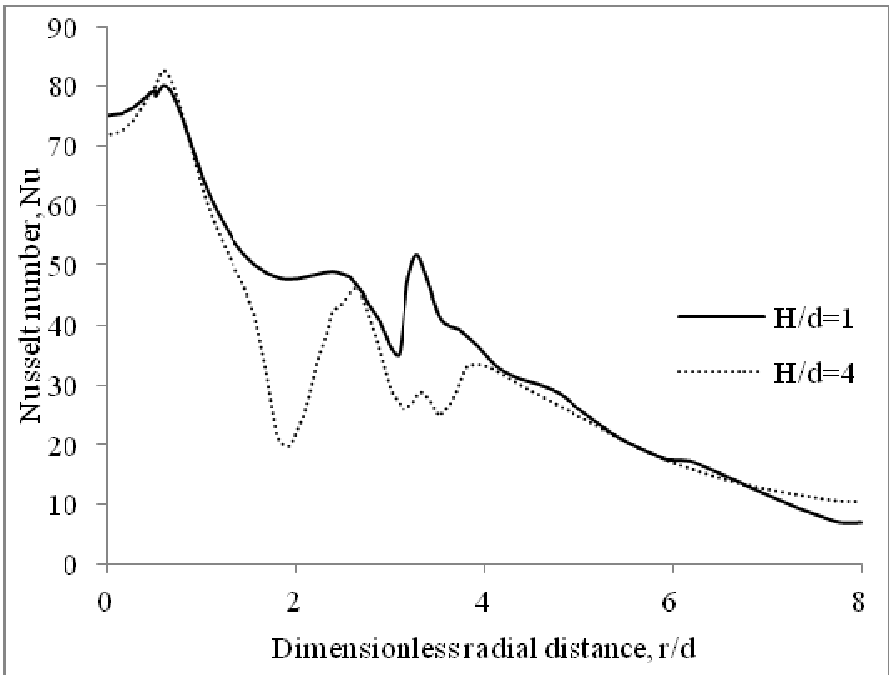

Fig. 16 Nusselt number distribution on line B-B; for $\mathrm{H} / \mathrm{d}=2$ and $\mathrm{q} "=3000 \mathrm{~W} / \mathrm{m}^{2} . \mathrm{K}$ 


\subsection{Comparison with single impinging jet}

A comparative CFD study is made between the two cases: shower head and a single jet. The dimensions for single jet are decided by considering equal mass flow rate for both the cases. Thus the shower head (five) jets of $10 \mathrm{~mm}$ diameter each are considered equivalent to single jet of diameter, $\mathrm{D}=22.36 \mathrm{~mm}$. The corresponding height will also be change. For example, $\mathrm{H} / \mathrm{d}=2$ implies the jet plate spacing of 20 $\mathrm{mm}$ for the shower head jet case and $44.72 \mathrm{~mm}$ for the single jet case.

Figure 17 shows the temperature distribution on top surface of the plate for single jet and shower head jet for $\mathrm{H} / \mathrm{d}=2, \mathrm{Re}=9500$ and $\mathrm{q} "=$ $3000 \mathrm{~W} / \mathrm{m}^{2} \mathrm{~K}$. It is observed that temperature reduces monotonically radially outward from stagnation point to the entire extent for single jet case. In case of shower head however the temperature distribution is more uniform because the wall jets and their interaction regions cover the whole surface of the impingement plate effectively.

Figure 18 shows the Nusselt number distribution on the impingement surface of the plate for $\mathrm{H} / \mathrm{d}=2, \mathrm{Re}=9500$ and $\mathrm{q} "=3000$ $\mathrm{W} / \mathrm{m}^{2} . \mathrm{K}$. In case of single jet the heat transfer rate is higher at the stagnation region and reduces radially outward. Whereas in case of shower head jet Nusselt number peaks form at the primary and secondary stagnation regions. This is because although temperature has a relatively uniform distribution, the heat flux distribution varies significantly throughout the plate surface.

Area weighted average heat transfer coefficient for the single jet is found to be $95 \mathrm{~W} / \mathrm{m}^{2} \mathrm{~K}$ and for shower head impinging jet is 101 $\mathrm{W} / \mathrm{m}^{2} \mathrm{~K}$ a difference of about $7 \%$. Specifically in the outer region of the plate the heat transfer rate lower for single jet, whereas in case of shower head heat transfer rate is almost uniform on the surface.

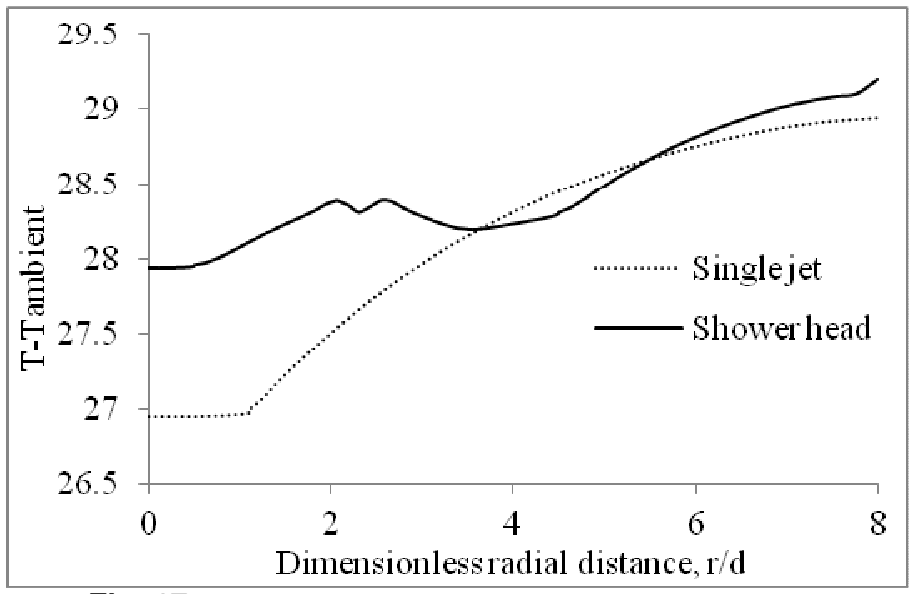

Fig. 17 Temperature distribution on impingement surface; for $\mathrm{H} / \mathrm{d}=2, \mathrm{Re}=9500$ and q" $=3000 \mathrm{~W} / \mathrm{m}^{2} . \mathrm{K}$

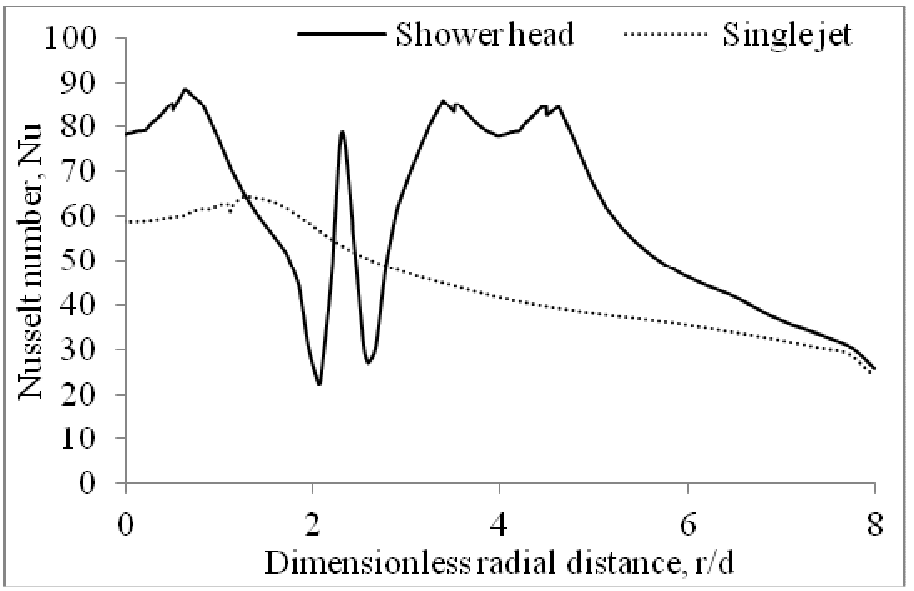

Fig. 18 Nusselt number distribution on impingement surface; for $\mathrm{H} / \mathrm{d}=2, \mathrm{Re}=9500$ and $\mathrm{q} "=3000 \mathrm{~W} / \mathrm{m}^{2} . \mathrm{K}$

\section{CONCLUSIONS}

Three dimensional conjugate heat transfer of flat circular disk is investigated with constant heat flux at the bottom surface and with a five-jet showerhead impinging on its top surface. The following conclusions are drawn from the study:

1. The good agreement between the measured temperature data within the plate and the conjugate heat transfer computational result obtained at the same locations prove the efficacy of the Shear Stress Transport (SST) $\kappa-\omega$ turbulence model for the chosen problem.

2. Nusselt number distribution on the plate is found to be independent of plate thickness. The difference in local Nusselt number is found to be maximum at second peak and is about $6 \%$ between impingement plates of $2.5 \mathrm{~mm}$ and $20 \mathrm{~mm}$ thickness. Whereas heat flux and temperature varies significantly across the solid plate due to flow structures and the plate thickness.

3. The features of the complex flow structure noticed due to the interactions of the central jet with the perimeter jets include (i) separation of the wall jets in the impingement plane (ii) the impingement of upwash fountain on the semi-confining upper plate for low $\mathrm{H} / \mathrm{d}$ values, (iii) formation of wall eddies in between counter rotating vortex and impingement wall.

4. The Nusselt number on the surface is found to vary in accordance with the flow structure. Peaks are noticed in the primary and secondary stagnation zones. It is observed that the local heat transfer rate is higher for lower $\mathrm{H} / \mathrm{d}$, whereas the area weighted average heat transfer rates are not affected much.

4. Heat transfer rate increases with the increase in Reynolds number. The Nusselt number peak at the nodal point of separation is smaller in magnitude than the peaks observed in the secondary stagnation region. Position of the secondary peak is found to be independent of Reynolds number.

5. Shower head jets provide uniform temperature distribution with higher heat transfer rate in comparison with the single jet for same mass flow rate. The area weighted average heat transfer coefficient is higher by about $7 \%$ for showerhead jet for equal mass flow rate.

\section{NOMENCLATURE}

$d$

$D$

$\mathrm{H}$

$h$

$k$

$l$

$\mathrm{L}$

$\mathrm{Nu}$

$\mathrm{p}$

$\operatorname{Pr}$

q"

$\mathrm{Re}$

$\mathrm{r}$

$\mathrm{R}$

$\mathrm{t}$

$\mathrm{T}$

$\mathrm{V}_{\mathrm{oe}}$

$\mathrm{V}_{\mathrm{z}}$

$\mathrm{V}_{\mathrm{r}}$

$\mathrm{V}_{\theta}$

$\mathrm{u}, \mathrm{v}, \mathrm{w}$
Jet orifice diameter for shower head jet, $\mathrm{m}$

Jet orifice diameter in case of single jet, $m$

Height of the orifice from plate, $m$

Heat transfer coefficient, $\mathrm{W} / \mathrm{m}^{2}$

Thermal conductivity (W/m-K)

Pitch circle diameter of the perimeter jet, $\mathrm{mm}$

Characteristic length of the impingement plate

Nusselt Number

Pressure, $\mathrm{Pa}$

Prandtl number

Wall heat flux, W/m²

Reynolds number, $\rho v d / \mu$

Radial co-ordinates

Radius of the plate, $m$

Thickness of the plate, $\mathrm{m}$

Temperature, $\mathrm{K}$

Orifice exit jet velocity, $\mathrm{m} / \mathrm{s}$

Vertical velocity, $\mathrm{m} / \mathrm{s}$

Radial velocity, $\mathrm{m} / \mathrm{s}$

Angular velocity, m/s

Greek Symbol

$\kappa \quad$ Turbulent kinetic energy, $\mathrm{m}^{2} / \mathrm{s}^{2}$

$\mu \quad$ Dynamic viscosity, $\mathrm{kg} / \mathrm{m}$-s 
$v \quad$ Kinematic viscosity, $\mathrm{m}^{2} / \mathrm{s}$

$\rho \quad$ Density, $\mathrm{kg} / \mathrm{m}^{3}$

$\omega \quad$ Specific dissipation rate, $1 / \mathrm{sec}$

Subscript

f $\quad$ Fluid

oe $\quad$ Orifice exit

S Solid

w Wall

Abbreviations

SDR Specific Dissipation Rate

SST Shear Stress Transport

TKE Turbulent Kinetic

\section{REFERENCES}

Aldabbagh, L.B.Y., and Sezai, I., 2002, "Numerical simulation of threedimensional laminar multiple impinging square jets," International Journal of Heat and Fluid Flow, Vol. 23, pp. 509-518. doi: 10.1016/ S0142 -727X(02)00141-8

Emmanuel B., L., Bernard, A., Bousgarbies, L., Dorignacj, E., and Vullierme, J., 2000, "Study of Several Impinging Jets," Journal of Thermal Science, Vol. 9, No. 3, pp. 217-223. doi: 10.1007/s11630-000-0054-6

Garimella, S. V. and Schroeder, V.P., 2001, "Local heat transfer distribution in confined multiple air jet impingement," ASME Journal of Electronic Packaging, Vol. 123, pp.165-172. doi: $10.1115 / 1.1371923$

Geers L.F.G., Tummers M.J., Bueninck, T.J., Hanjalic., K., 2008, "Heat transfer correlation for hexagonal and in-line arrays of impinging jets," International Journal of Heat and Mass Transfer, Vol. 51, 5389-5399. doi:10.1016/j.ijheatmasstransfer.2008.01.035

Huber, A.M., and Viskanta, R., 1994, "Comparison of convective heat transfer to perimeter and centre jets in a confined, impinging array of axi-symmetric air jets," International journal of Heat and Mass Transfer, Vol. 37, pp. 3025-3030.

doi:10.1016/0017-9310(94)90340-9

Jambunathan, K., Lai, E., Moss, M.A., and Button, B.L., 1993, "A review of heat transfer data for single circular impingement," International Journal of Heat Fluid Flow, Vol. 13, No. 2, pp. 106-115. doi:10.1016/0142-727X(92)90017-4

Katti, V., and Prabhu, S.V., 2007, "Experimental study and theoretical analysis of local heat transfer distribution between smooth flat surface and impinging air jet from a circular straight pipe nozzle," International Journal of Heat and Mass Transfer, Vol. 51, pp. 4480-4495. doi: 10.1007/s00231-010-0716-1
Luikov, A. V., 1973, "Conjugate convective heat transfer problems," International Journal of Heat and Mass Transfer, Vol.17 pp. 257265. doi:10.1016/0017-9310(74)90087-8

Martin, H., 1977, "Heat and mass transfer between impinging gas jets and solid surface," Advances in Heat Transfer, Vol. 13, pp. 1-60. doi: 00/1977

Naitoh, T., Osamu, B., and Hideo Y., 2001, "Longitudinal vortex structure in the flow field produced by a vortex ring impinging on a plate," Fluid Dynamics Research, Vol. 16, No. 4, pp. 61-74. doi: 10.1016/S0169-5983(00)00016-2.

O’Donovan, T. S., and Darina B. M., 2007, "Jet impingement heat transfer - Part 2: A temporal investigation of heat transfer and local fluid velocities," International Journal of Heat and Mass Transfer, Vol. 50, pp. 3302-3314.

doi:10.1016/j.ijheatmasstransfer.2007.01.047

Panda, R. K., Sreekala P., and Prasad, B.V.S.S.S., 2010, "Computational And Experimental Study Of Conjugate Heat Transfer From A Flat Plate With Shower Head Impinging Jets," ASMEInternational Heat Transfer Conference (IHTC-14), IHTC14-23188, Washington D.C., USA.

Prasad, B.V.S.S.S., and Sarkar, S. D., 1993, "Conjugate laminar forced convection from a flat plate with imposed pressure gradient," ASME Journal of Heat Transfer, Vol. 115, pp. 469-472. doi: $05 / 1993$

Ramakumar B.V.N. and Prasad, B.V.S.S.S., 2008, "Computational flow and heat transfer of a row of circular jets impinging on a concave surface," Heat and Mass Transfer, Volume 44, pp 667-678. doi: 10.1007/s00231-007-0274-3

Sagot, B., Antonini, G., Christgen, A. and F. Buron, 2007, "Jet impingement heat transfer on a flat plate at a constant wall temperature," International Journal of Thermal Sciences, Vol. 47, pp.1610-1619.

doi:10.1016/j.ijthermalsci.2007.10.020

Siba, E. A., Ganesa-Pillai, M., Harris, K. T. and Haji-Sheikh, A., 2003, "Heat transfer in a high turbulence air jet impinging over a flat circular disk," ASME Journal of Heat Transfer, Vol. 125, pp. 257-265. doi:10.1115/1.1469523

Viskanta, R., 1993, "Heat Transfer to Impinging Isothermal Gas and Flame Jets," Experimental Thermal and Fluid Science, Vol. 6, pp.111134. doi:10.1016/0894-1777(93)90022-B

Yang, Y-T. and Tsai, S.Y., 2007, "Numerical study of transient conjugate heat transfer of a turbulent impinging jet," International Journal of Heat and Mass Transfer, Vol. 50, pp. 799-807. doi:10.1016/j.ijheatmasstransfer.2006.08.022 